\title{
Molecular Phylogenetic Relationships of the Brown Leaf Rust Fungi on Wheat, Rye, and Other Grasses
}

Miao Liu and Les J. Szabo, United States Department of Agriculture-Agricultural Research Service (USDA-ARS) Cereal Disease Laboratory, St. Paul, MN 55108; Sarah Hambleton, Biodiversity (Mycology and Botany), Eastern Cereal and Oilseed Research Centre, Agriculture and Agri-Food Canada, Ottawa, ONT K1A 0C6, Canada; Yehoshua Anikster, Institute for Cereal Crops Improvement, George S. Wise Faculty of Life Science, Tel Aviv University, Tel Aviv 69978, Israel; and James A. Kolmer, USDA-ARS Cereal Disease Laboratory, St. Paul

\begin{abstract}
Liu, M., Szabo, L. J., Hambleton, S., Anikster, Y., and Kolmer, J. A. 2013. Molecular phylogenetic relationships of the brown leaf rust fungi on wheat, rye, and other grasses. Plant Dis. 97:1408-1417.

The classification of brown leaf rust fungi (Puccinia recondita complex and allied species) on wheat (Triticum aestivum), rye (Secale cereale), and other grasses in the family Poaceae has experienced a long history of controversy and uncertainty due to the reduced morphological characteristics available for taxonomy and difficulty of conducting interfertility experiments. However, because these are pathogens on important crops, it is important to clarify the species delimitations reflecting the natural lineages. In this study, phylogenetic analyses were conducted with DNA sequence data from the ribosomal DNA internal transcribed spacer region and elongation factor $1-\alpha$ to

elucidate this species complex. Three phylogenetic lineages were recovered within the complex of rye leaf rust fungi, $P$. recondita sensu stricto, which is congruent with existing classifications based on DNA content, sexual compatibility, and morphological studies. The brown leaf rust fungus on wheat ( $P$. triticina) grouped with the related species P. persistens on Elymus repens and E. intermedia as a strongly supported clade. Collections on other Elymus spp. were separated into six clades. Based on the phylogenetic affinities of nine type specimens and aecial host associations, potential taxonomic names were evaluated for selected lineages.
\end{abstract}

The classification and nomenclature of brown leaf rust (or brown rust) fungi on wheat (Triticum aestivum L.), rye (Secale cereale L.), and various grasses in the family Poaceae has historically been contentious. A rust pathogen on wheat was first described as a form species by De Candolle in 1815 (10) with the name Uredo rubigo-vera DC. In 1884, Winter moved the taxon to the genus Puccinia; that is, Puccinia rubigo-vera (DC) G. Winter (48). From the late 1800 s to the early 1900 s, it was generally accepted by many mycologists in Europe and North America that only one species consisting of leaf rust fungi on wheat, rye, barley, and various grasses was present (namely, P. rubigo-vera) that produced globoid urediniospores and long, covered telia (48) (Fig. 1). The aecial hosts were generally expected to be in the family Boraginaceae due to the successful infections obtained by de Bary $(9,12,18)$ using the leaf rust fungus of rye to inoculate Anchusa officinalis L and A. [Lycopsis] arvensis. When Plowright (34) described a new species in 1885 with the name P. perplexans Plowr. on Alopecurus pratensis alternating with Ranunculus acris (family Ranunculaceae), it was noted that more than one species could be recognized in brown leaf rust fungi on various grasses. Afterward, numerous species were recognized and named based on the host specialization (28), and the classification of these cereal or grass leaf rust fungi continued to be revised from time to time $(3,4,7,14,15,28)$.

Landmark revisions took place as follows. Eriksson and Henning (13-15) in 1894 and 1899 recognized multiple species previously grouped in P. rubigo-vera; that is, P. agropyrina Erikss. on

Corresponding author: J. A. Kolmer,

E-mail: Jim.Kolmer@ARS.USDA.gov

Accepted for publication 1 June 2013.

http://dx.doi.org/10.1094/PDIS-02-13-0152-RE

This article is in the public domain and not copyrightable. It may be freely reprinted with customary crediting of the source. The American Phytopathological Society, 2013.
Agropyron spp., P. bromina Erikss. on Bromus spp., P. dispersa Erikss. \& Henning on rye (S. cereale) with alternate hosts in Anchusa spp. (family Boraginaceae); P. glumarum (J.C. Schmidt) on Triticum spp. and other grasses; P. holcina Erikss. on Holcus lanatus and H. mollis; P. simplex (Körn) Erikss. \& Henning on Hordeum spp.; P. triseti Erikss. on Trisetum flavescens, and P. triticina Erikss. on Triticum aestivum. In contrast, Arthur and Fromme (4) combined all the forms of $P$. rubigo-vera with aecial hosts in the family Ranunculaceae into one species under the name of $\mathrm{Di}$ caeoma clematidis (DC) Arthur, and designated other species, such as D. apocrypta (Ellis \& Tracy) Kuntze on Agropyron, Elymus, and Sitanion spp., with aecial hosts in the families Hydrophyllaceae and Boraginaceae; D. asperifolii (Pers.) Kuntze on S. cereale, with

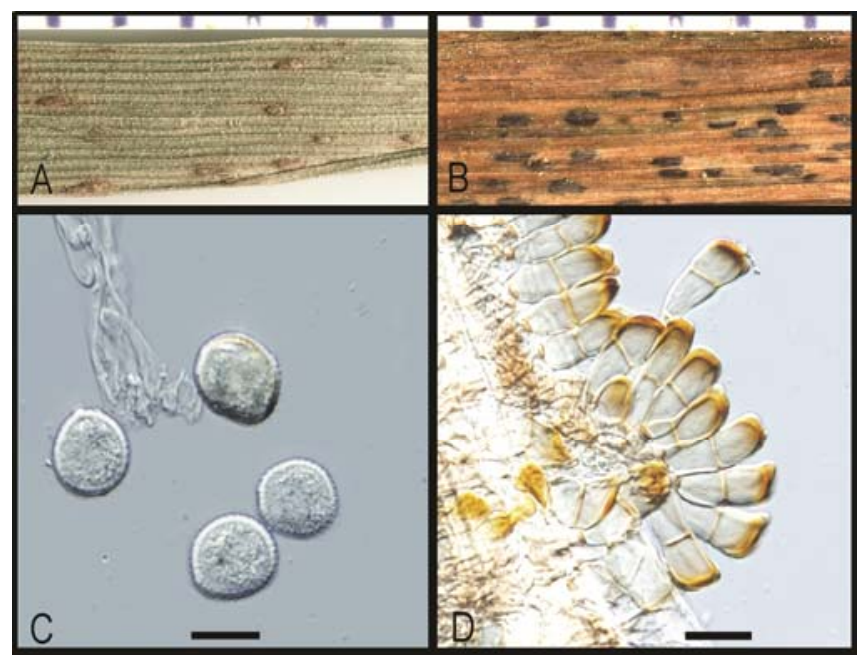

Fig. 1. Puccinia recondita on Hordeum jubatum (uredinial stage, PUR N1436) and Elymus hystrix (telial stage, PUR N5336). A, Cinnamon-brown uredinia on adaxial leaf surface; B, blackish-brown-covered telia on abaxial leaf surface; C, globoid urediniospores; D, clavate teliospores. Scale bars $=20 \mu \mathrm{m}$ for $\mathrm{C}$ and $\mathrm{D} ; 1$ unit $=1$ $\mathrm{mm}$ for $\mathrm{A}$ and $\mathrm{B}$. 
Anchusa officinalis as aecial host; and D. impatientis (Schw.) Arthur on Agrostis, Cinna, Elymus, Hordeum, and Hystrix spp., with Impatiens spp. as aecial host.

Mains disagreed with earlier authors, and recommended placement of all recognized species back into one species under the name $P$. rubigo-vera, listing 32 names as synonyms, and instead separated the species into 56 formae speciales according to infection on various host species (28). Arthur accepted the gramineous leaf rust fungi as one species, $P$. rubigo-vera, with six varieties (3). Cummins and Caldwell proposed $P$. recondita Roberge ex Desm. 1857 as the legitimate binomial for the brown leaf rust fungi on cereals and grasses (8). Cummins (7) treated brown leaf rust fungi as one species under the name $P$. recondita and listed 51 binomials as synonyms. This broad species concept was prevalent in North America, although Savile (39) accepted the taxon on wheat as a distinct species, P. triticina designated by Eriksson (14).

In Europe, Urban and Marková $(30,45,46)$ accepted six species to replace the $P$. recondita complex (i.e., $P$. bromina, $P$. elymi Westend., $P$. perplexans, $P$. persistens Plowr., $P$. recondita sensu stricto, and $P$. schismi Bubák. They considered $P$. recondita sensu stricto as the species that infects Aegilops, Secale, and Triticum spp. with aecial hosts in the family Boraginaceae, and P. persistens as the species that is prevalent on various gramineous uredinial or telial hosts with aecial hosts in the family Ranunculaceae (except $P$. persistens subsp. agropyri that can occur on the family Boraginaceae in Europe). The wheat leaf rust fungus ( $P$. triticina) was considered as a subspecies of $P$. persistens. Based on DNA content, morphology, and interfertility, Anikster et al. (1) observed two groups in a collection of leaf rust fungi from cultivated wheat, wild emmer wheat (Triticum turgidum subsp. dicoccoides), and Aegilops speltoides Tausch from Israel. Group I, equivalent to Eriksson's $P$. triticina with virulence to common wheat and durum wheat, had alternate hosts in Thalictrum L. Group II with virulence to Aegilops spp. and T. turgidum (durum wheat) and alternate hosts in the family Boraginaceae was equivalent to a subset of $P$. recondita sensu Cummins (7).

The grass genus Elymus L., commonly known as wild rye or wheat grasses, has served as a rich germplasm resource for disease and pest resistance for wheat breeding (19) and is also a major host for brown leaf rusts. The identities of the brown leaf rusts on wheat, rye, Aegilops spp., and Elymus spp. have not been established in a phylogenetic context. In the present study, we explored the phylogenetic lineages in the brown leaf rusts on these selected hosts using DNA sequence analyses. In particular, we focused on the following questions: (i) did these lineages correspond to the taxonomic concepts in current use for cereal rust fungi, with specific emphasis on $P$. recondita, $P$. triticina, and $P$. persistens; and (ii) did the brown leaf rusts on Elymus spp. form a monophyletic phylogenetic group.

\section{Materials and Methods}

Leaf rust collections. Thirty-one $P$. recondita specimens on genus Elymus (the host of some specimens were labeled as Agropyron, Leymus, and Hystrix; Table 1) were obtained from herbaria at the J. F. Bell Museum of Natural History, University of Minnesota, St. Paul (MIN); Purdue University, West Lafayette, IN (PUR); and the Botanischer Garten und Botanisches Museum Berlin-Dahlem, Berlin, Germany (B). In all, 33 type specimens were obtained from the Swedish Museum of Natural History, Stockholm, Sweden (S, 1 sample); Royal Botanic Gardens, Kew, England, UK (K, 1 sample); and PUR (31 samples). Based on the condition of the specimens, only nine type specimens were selected for DNA extraction for species $P$. actaeae-elymi Mayor, $P$. agropyrina, $P$. dactylidina Bubák., $P$. perplexans, $P$. persistens, $P$. recondita, $P$. subalpina Lagerh., $P$. triticina, and $P$. wyomensis Arthur. Seventy-four additional specimens or samples were obtained from herbaria or collected (purified as single uredinium isolates for $P$. triticina) and kept in individual labs for the following species: $P$. arrhenathericola E. Fisch., P. bromina, P. coronata Corda., P. coronatibrevispora M. Liu \& Hambl., P. coronati-calamagrostidis M. Liu
\& Hambl., P. coronati-hordei M. Liu \& Hambl., P. coronatilongispora M. Liu \& Hambl., P. gansensis M. Liu \& Hambl., P. graminis Pers., P. holcina, $P$. hordei G. H. Otth., P. persistens, $P$. poarum Nielsen, P. pseudostriiforimis M. Abbasi., Hedjar. \& M. Scholler, $P$. recondita (on hosts other than Elymus spp.), P. striiformis Westend., P. striiformoides M. Abbasi., Hedjar. \& M. Scholler, and P. triticina. Uromyces dactylidis G.H. Otth. was selected to serve as outgroup taxon based on previous studies (25-27). Voucher numbers, hosts, origins, and GenBank accession numbers are listed in Table 1. Sequences for some specimens were determined in previous studies by M. Liu and S. Hambleton, as indicated in Table 1.

Genomic DNA extraction. Small pieces of infected plant material, $1-4.33$ by $0.1-0.8 \mathrm{~cm}$, or urediniospores were used for genomic DNA extraction. Plant materials were surface-sterilized by gentle wiping using a Kimwipe (KimTech, Inc.) sprayed with $75 \%$ ethanol. The cleaned infected plant material or pure uredinial spores were ground with $25 \mathrm{mg}$ of glass beads and a small amount of diatomaceous earth with a FastPrep-24 shaker (M. P. Biomedicals), followed by DNA extraction using OmniPrep (GBioSciences) as per the manufacturer's instructions. RNase was added to the final DNA solution with no further precipitation.

Polymerase chain reaction amplification and sequencing. For aging herbarium specimen samples, the PreCR Repair Mix (New England BioLabs) was used to attempt DNA repair before polymerase chain reaction (PCR) amplifications. The nuclear ribosomal DNA (rDNA) region, including 5.8S and internal transcribed spacer 2 (ITS2), was amplified and sequenced using primers Rust2inv and ITS4, using methods described by Liu and Hambleton (25). A fragment of elongation factor 1- $\alpha$ (EF1- $\alpha$ ) was amplified using forward primer EF1-Pt1-113f (ATCCTCACAGCGG TAAATCCACCA) and reverse primer EF1-Pt1-904r (ACACAC CTCTTTGGTCCATCCCTT) or, alternatively, using forward primer EF1-Pt2-032f (TCGTCATTGGACACGTCGATTCGT) and reverse primer EF1-Pt2-552r (AGTACCGGCAGCAATGATGAG GAT), depending on whichever pair was successful. These primers were designed based on the EF1- $\alpha$ DNA sequences extracted from two Puccinia whole genome sequences ( $P$. graminis and $P$. triticina) available on the Broad Institute website (http://www.broad institute.org/annotation/genome/puccinia_group/MultiHome.html). PCR was performed in $10-\mu \mathrm{l}$ reactions containing $0.1 \mathrm{mM}$ dNTPs, 2.0 pmol of each primer, $1 \mu$ l 10× Titanium Taq buffer, and $0.1 \mu \mathrm{l}$ of $50 \times$ Titanium Taq DNA Polymerase (BD Biosciences). Thermal cycling conditions were set as $95^{\circ} \mathrm{C}$ for 3 min; followed by 38 cycles of $95^{\circ} \mathrm{C}$ for $30 \mathrm{~s}, 63^{\circ} \mathrm{C}$ for $20 \mathrm{~s}$, and $72^{\circ} \mathrm{C}$ for $2 \mathrm{~min}$; followed by a final extension of $72^{\circ} \mathrm{C}$ for $8 \mathrm{~min}$. PCR products were cleaned by using ExoSAP-IT (USB Products Affymetrix, Inc.) and sequenced using PCR primers through the BioMedical Genomics Center services (University of Minnesota, St. Paul).

Phylogenetic analyses. Sequences were edited using Sequencher 4.7 (Gene Codes Corporation) and compiled using BioEdit Sequence Alignment Editor 7.0.5.3 (17). The compiled sequences were submitted to a web server (http://mafft.cbrc.jp/ alignment/server/index.html) for alignment by MAFFT ver.5 (20), and the model FFT-NS-I (iterative refinement method) was selected. The sequence alignments were manually adjusted to correct obvious misalignments by the computer algorithm.

Phylogenetic analyses were conducted for each locus separately and for the combined data set. For parsimony analysis in PAUP* $4.0 \mathrm{~b} 10$ (42), heuristic searches with random stepwise addition of 100 replicates and tree bisection-reconnection branch swapping were conducted. A limit of 1,000,000 rearrangements was set for each replicate. Bootstrapping analyses were set with 1,000 replicates with full heuristic search of random stepwise addition of 10 replicates and limit of 10,000 rearrangements per replicate.

Bayesian analysis was conducted using MrBayes 3.2.0 (37), (http://www.mrbayes.net) with different DNA substitution models estimated with Modeltest 3.6 (36). For the 5.8S-ITS2 locus, state frequencies $=0.3750,0.1530,0.1477$, and 0.3243 ; substitution 
rates $=2.3889,2.5075,1.9954,0.2455,3.9896$, and 1.0000; proportion of invariable sites $=0.3884$; and $\gamma$ distribution shape parameter $=0.4321$. For $E F 1-\alpha$, state frequencies $=0.2484,0.3098$, 0.1885 , and 0.2533 ; substitution rates $=1.0000,2.7944,1.0000$, $1.0000,4.0323$, and 1.0000; proportion of invariable sites $=$
0.4928 ; and $\gamma$ distribution shape parameter $=3.0565$. Four chains of 5,000,000 Markov chain Monte Carlo generations were run. The first 5,000 trees were discarded (burn-in) and the remaining trees were spooled to PAUP* to obtain posterior probabilities based on the $50 \%$ majority-rule consensus.

Table 1. Fungal (Puccinia spp.) isolates, hosts, origins, and GenBank accession numbers for internal transcribed spacer (ITS) of the ribosomal and elongation factor $(\mathrm{EF})$

\begin{tabular}{|c|c|c|c|c|c|}
\hline Species & Voucher numbers $^{\mathrm{a}}$ & Hosts $^{\text {b }}$ & Origins & ITS & $\mathbf{E F}^{\mathbf{c}}$ \\
\hline Puccinia actaeae-elymi $i^{\mathrm{d}, \mathrm{e}}$ & PUR F4124 (T) & Elymus europaeus & Switzerland & JX533543 & JX533485 \\
\hline P. agropyrina ${ }^{\mathrm{d}, \mathrm{e}}$ & PUR F4069 (T) & E. repens & Sweden & JX533542 & $\mathrm{n} / \mathrm{a}$ \\
\hline P. arrhenathericola ${ }^{\mathrm{d}, \mathrm{e}}$ & HSZ 0658 & Arrhenatherum elatius & Slovakia & JX533581 & JX533452 \\
\hline P. bromina $\mathrm{d}^{\mathrm{e}} \mathrm{e}$ & HSZ 0660 & Bromus sterilis & Slovakia & JX533582 & JX533453 \\
\hline P. bromina ${ }^{\mathrm{e}}$ & $\mathrm{K}(\mathrm{M}): 107810$ & B. sterilis & England & JX533578 & $\mathrm{n} / \mathrm{a}$ \\
\hline P. coronata var. avenae f. sp. avenae & BR 8665 & $\begin{array}{l}\text { Agropyron coninum } \\
\text { (syn. of } \text { E. caninus) }\end{array}$ & Belgium & HM131278f & $\mathrm{n} / \mathrm{a}$ \\
\hline P. coronata var. avenae & & & & & \\
\hline f. sp. graminicola & PUR N 1252 & Holcus lanatus & United States & HM131251 ${ }^{\mathrm{f}}$ & $\mathrm{n} / \mathrm{a}$ \\
\hline P. coronata var. coronata & (S) Reg.nr.F46266 & Frangula almus & Sweden & HM131310 f & $\mathrm{n} / \mathrm{a}$ \\
\hline P. coronati-agrostidis & PUR N1029 & Agrostis sp. & Canada & HM131327f & $\mathrm{n} / \mathrm{a}$ \\
\hline P. coronati-brevispora & DAOM 240183 & B. inermis & Canada & HM131237f & $\mathrm{n} / \mathrm{a}$ \\
\hline P. coronati-calamagrostidis & PUR N2268 & Calamagrostis canadensis & United States & HM131308f & $\mathrm{n} / \mathrm{a}$ \\
\hline P. coronati-hordei & DAOM 183691 & E. repens & Canada & HM057138f & $\mathrm{n} / \mathrm{a}$ \\
\hline P. coronati-hordei & PUR 89857 & Hordeum vulgare & United States & HM131225 & $\mathrm{n} / \mathrm{a}$ \\
\hline P. coronati-longispora & PRC 196 & B. erectus & Czech Rep. & HM131232f & $\mathrm{n} / \mathrm{a}$ \\
\hline P. dactylitin $a^{\mathrm{d}, \mathrm{e}}$ & PUR F15426 (T) & Dactylis glomerata & Czech Rep. & JX533546 & $\mathrm{n} / \mathrm{a}$ \\
\hline P. gansensis & DAOM 240065 & Achnatherum inebrians & China & HM057115g & $\mathrm{n} / \mathrm{a}$ \\
\hline P. graminis & TF NZ5 03 & Avena sp. & New Zealand & HQ012437 h & $\mathrm{n} / \mathrm{a}$ \\
\hline$P$. graminis & BPI 803290 & $\begin{array}{l}\text { Elytrigia repens } \\
\text { (syn. of Elymus repens) }\end{array}$ & China & HM131359f & $\mathrm{n} / \mathrm{a}$ \\
\hline P. graminis & PUR 66554 & Oryzopsis exigua & United States & HM131358f & $\mathrm{n} / \mathrm{a}$ \\
\hline P. graminis & PUR N1125 & Secale cereale & Germany & HQ012443 & $\mathrm{n} / \mathrm{a}$ \\
\hline P. graminis & TF SA50 04 & Triticum aestivum & South Africa & HQ012444h & $\mathrm{n} / \mathrm{a}$ \\
\hline P. holcina ${ }^{\mathrm{d}, \mathrm{e}}$ & HSZ 0681 & Holcus lanatus & Czech Rep. & DQ512999 & JX533454 \\
\hline P. hordei & BR 6861233 & Hordeum тигіпит & Belgium & HQ012448 & $\mathrm{n} / \mathrm{a}$ \\
\hline P. hordei & $\mathrm{K}(\mathrm{M}): 78624$ & H. murinum & England & HQ012449 & $\mathrm{n} / \mathrm{a}$ \\
\hline P. perplexans $\mathrm{s}^{\mathrm{d}, \mathrm{e}}$ & PUR F4086 (T) & Alopecurus pratensis & England & JX533545 & JX533487 \\
\hline P. persistens ${ }^{\mathrm{e}}$ & K(M): 78119 & Agropyron repens (syn. of E. repens) & England & JX533586 & $\mathrm{n} / \mathrm{a}$ \\
\hline P. persistens $\mathrm{d}^{\mathrm{d}}$ & HSZ 0688 & E. intermedia & Czech Rep. & JX533517 & JX533459 \\
\hline P. persistens ${ }^{\mathrm{d}}$ & HSZ 0691 & E. intermedia & Czech Rep. & JX533519 & JX533461 \\
\hline P. persistens ${ }^{\mathrm{d}}$ & HSZ 0696 & E. intermedia & Czech Rep. & JX533521 & JX533463 \\
\hline P. persistens ${ }^{\mathrm{d}}$ & ISR 3563 & E. intermedia & Czech Rep. & JX533511 & JX533464 \\
\hline P. persistens $\mathrm{d}^{\mathrm{d}}$ & ISR 3675 & E. intermedia & Czech Rep. & JX533512 & JX533465 \\
\hline P. persistens $\mathrm{d}$ & HSZ 0686 & E. repens & Czech Rep. & JX533515 & JX533457 \\
\hline P. persistens $\mathrm{d}^{\mathrm{d}}$ & HSZ 0687 & E. repens & Czech Rep. & JX533516 & JX533458 \\
\hline P. persistens ${ }^{\mathrm{d}}$ & HSZ 0689 & E. repens & Sweden & JX533518 & JX533460 \\
\hline P. persistens ${ }^{\mathrm{d}}$ & HSZ 0695 & E. repens & Czech Rep. & JX533520 & JX533462 \\
\hline P. persistens ${ }^{\mathrm{d}}$ & ISR 6134 & E. repens & England & JX533513 & JX533466 \\
\hline P. persistens $\mathrm{d}^{\mathrm{d}}$ & ISR 6140 & E. repens & England & JX533514 & JX533467 \\
\hline P. persistens & DAOM 162167 & Thalictrum flavum & Finland & JX533585 & $\mathrm{n} / \mathrm{a}$ \\
\hline P. persistens & HSZ 0419 & T. тіпиs & Slovakia & JX533579 & $\mathrm{n} / \mathrm{a}$ \\
\hline P. persistens & HSZ 0692 & T. minus & Czech Rep. & JX533580 & $\mathrm{n} / \mathrm{a}$ \\
\hline P. persistens ${ }^{\mathrm{d}}$ & $\mathrm{K}(\mathrm{M}): 169739(\mathrm{~T})$ & Triticum repens & United Kingdom & JX533510 & $\mathrm{n} / \mathrm{a}$ \\
\hline P. poarum & DAOM 240188 & Tussilago farfara & Canada & HM057150g & $\mathrm{n} / \mathrm{a}$ \\
\hline P. pseudostriiformis & PUR N5354 & Poa nemoralis & United States & HM057134g & $\mathrm{n} / \mathrm{a}$ \\
\hline Puccinia pseudostriiformis & DAOM 220657 & Poa pratensis & Canada & HM057113 & $\mathrm{n} / \mathrm{a}$ \\
\hline Puccinia recondita $^{\mathrm{d}}$ & ISR 77069 & Aegilops longissima & Israel & JX533537 & JX533481 \\
\hline P. recondita ${ }^{\mathrm{d}}$ & ISR 9382 & A. longissima & Israel & JX533535 & JX533479 \\
\hline P. recondita ${ }^{\mathrm{d}}$ & ISR 9003 & A. ovata & Israel & JX533530 & JX533474 \\
\hline$P$. recondita ${ }^{\mathrm{d}}$ & ISR 9284 & A. ovata & Israel & JX533531 & JX533475 \\
\hline P. recondita ${ }^{\mathrm{d}}$ & ISR 9295 & A. ovata & Israel & JX533532 & JX533476 \\
\hline
\end{tabular}

${ }^{a}$ B: Botanischer Garten und Botanisches Museum Berlin-Dahlem, Zentraleinrichtung der Freien Universität Berlin, Berlin; BP: Hungarian Natural History Museum, Budapest; BPI: U.S. National Fungus Collections, Beltsville, MD; BR: National Botanic Garden of Belgium, Meise, Belgium; DAOM: Agriculture and Agri-Food Canada, Ottawa, ONT, Canada; HSZ: specimens provided by Dr. J. Marková and DNA samples held by LSZ lab; ISR: specimens held by Y. Anikster; K(M): Royal Botanic Gardens, Kew, England, UK; MIN: J. F. Bell Museum of Natural History, University of Minnesota, St. Paul; PRC: Charles University in Prague, Praha, Czech Republic; Pt: samples held by J. Kolmer as DNA voucher only; PUR: Purdue University, West Lafayette, IN; RS: one sample held by S. Hambleton as DNA voucher only; S: Swedish Museum of Natural History, Stockholm; and TF: one pure spore sample provided by Dr. T. Fetch. (T) indicates the specimen is the type specimen.

${ }^{\mathrm{b}}$ Hosts are listed as original identification on the specimen package.

c Abbreviation: $\mathrm{n} / \mathrm{a}=$ not applicable.

d Taxa included in analyses for the combined data set.

${ }^{\text {e }}$ Species belong to $P$. recondita sensu lato complex according to Cummins (7).

${ }^{\mathrm{f}}$ Sequences published by Liu and Hambleton (26).

${ }^{\mathrm{g}}$ Sequences published by Liu and Hambleton (25).

${ }^{\mathrm{h}}$ Sequences published by Liu and Hambleton (27). 


\section{Results}

DNA amplification and sequencing. Because of the ease of amplifying rDNA, more rDNA sequences than EF1- $\alpha$ sequences were successfully obtained for a large number of $P u c$ cinia spp. Among the 114 Puccinia sp. samples, amplification of the fragment of 5.8S-ITS2 was attempted for 86 samples, and 82 were successfully amplified, including 9 type specimens. Twenty-eight sequences of reference species from previous studies were also used (25-27). As a single-copy gene, EF1- $\alpha$ had a lower success rate for amplification, and 58 sequences out of 86 samples were obtained, including 4 type

Table 1. (continued from preceding page)

\begin{tabular}{|c|c|c|c|c|c|}
\hline Species & Voucher numbers $^{a}$ & Hosts $^{b}$ & Origins & ITS & $\mathbf{E F}^{\mathbf{c}}$ \\
\hline$P$. recondita ${ }^{\mathrm{d}}$ & ISR 99018 & A. ovata & Israel & JX533539 & JX533483 \\
\hline$P$. recondita ${ }^{\mathrm{d}}$ & ISR 9350 & A. variabilis & Israel & JX533533 & JX533477 \\
\hline$P$. recondita ${ }^{\mathrm{d}}$ & ISR 9357 & A. variabilis & Israel & JX533534 & JX533478 \\
\hline$P$. recondita ${ }^{\mathrm{d}}$ & ISR 99128 & A. variabilis & Israel & JX533540 & JX533484 \\
\hline$P$. recondita ${ }^{\mathrm{d}}$ & PUR 55737 & Agropyron trachycaulum & United States & JX533563 & $\mathrm{n} / \mathrm{a}$ \\
\hline$P$. recondita ${ }^{\mathrm{d}}$ & MIN 512350 & B. ciliatus & United States & JX533522 & $\mathrm{n} / \mathrm{a}$ \\
\hline$P$. recondita ${ }^{\mathrm{d}}$ & HSZ 0694 & Clematis sp. & Slovakia & JX533583 & JX533455 \\
\hline$P$. recondita & PUR 65476 & E. albican & United States & JX533550 & $\mathrm{n} / \mathrm{a}$ \\
\hline$P$. recondita ${ }^{\mathrm{d}}$ & PUR 24872 & E. alskanus & Canada & JX533549 & $\mathrm{n} / \mathrm{a}$ \\
\hline$P$. recondita ${ }^{\mathrm{d}}$ & PUR 55732 & E. alskanus & United States & JX533548 & JX533489 \\
\hline$P$. recondita & PUR 59584 & E. arizonicus & Mexico & JX533553 & $\mathrm{n} / \mathrm{a}$ \\
\hline$P$. recondita ${ }^{\mathrm{d}}$ & MIN 870591 & E. canadensis & United States & JX533525 & JX533470 \\
\hline$P$. recondita & PUR 25161 & E. canadensis & Canada & JX533554 & $\mathrm{n} / \mathrm{a}$ \\
\hline P. recondita & PUR N1447 & E. canadensis & United States & JX533589 & $\mathrm{n} / \mathrm{a}$ \\
\hline P. recondita & B 700012410 & E. caninus & Romania & HM131362 f & $\mathrm{n} / \mathrm{a}$ \\
\hline$P$. recondita ${ }^{\mathrm{d}}$ & PUR N76 & E. caninus & Finland & JX533555 & JX533490 \\
\hline$P$. recondita ${ }^{\mathrm{d}}$ & PUR 59754 & E. cinerus & Canada & JX533556 & JX533491 \\
\hline$P$. recondita & PUR 25210 & E. condensatus & United States & JX533557 & $\mathrm{n} / \mathrm{a}$ \\
\hline P. recondita & PUR F15636 & E. dahuricus & Japan & JX533558 & $\mathrm{n} / \mathrm{a}$ \\
\hline$P$. recondita ${ }^{\mathrm{d}}$ & PUR 59448 & E. glaucus & United States & JX533560 & JX533493 \\
\hline$P$. recondita ${ }^{\mathrm{d}}$ & PUR 59756 & E. glaucus & Canada & JX533559 & JX533492 \\
\hline$P$. recondita & PUR N1240 & E. hystrix & United States & JX533588 & $\mathrm{n} / \mathrm{a}$ \\
\hline P. recondita & PUR F17100 & E. repens & Bulgaria & JX533561 & $\mathrm{n} / \mathrm{a}$ \\
\hline$P$. recondita & PUR N1022 & E. repens & Canada & JX533587 & $\mathrm{n} / \mathrm{a}$ \\
\hline$P$. recondita ${ }^{\mathrm{d}}$ & PUR F16132 & E. semicostatus & Japan & $\mathrm{n} / \mathrm{a}$ & JX533494 \\
\hline$P$. recondita & PUR 25211 & E. submuticus & Canada & JX533562 & $\mathrm{n} / \mathrm{a}$ \\
\hline$P$. recondita ${ }^{\mathrm{d}}$ & PUR N1443 & E. villosus & United States & JX533564 & JX533495 \\
\hline$P$. recondita ${ }^{\mathrm{d}}$ & MIN 870593 & E. violosus & United States & JX533526 & JX533471 \\
\hline$P$. recondita ${ }^{\mathrm{d}}$ & MIN 512787 & E. virginicus & United States & JX533523 & JX533468 \\
\hline$P$. recondita & MIN 870607 & E. virginicus & United States & JX533527 & $\mathrm{n} / \mathrm{a}$ \\
\hline$P$. recondita ${ }^{\mathrm{d}}$ & MIN 870610 & E. virginicus & United States & JX533528 & JX533472 \\
\hline$P$. recondita ${ }^{\mathrm{d}}$ & MIN 513209 & Hystrix patula (syn. of E. hystrix) & United States & JX533524 & JX533469 \\
\hline$P$. recondita ${ }^{\mathrm{d}}$ & HSZ 0764 & Impatiens $c f$. pallida & United States & JX533584 & JX533456 \\
\hline$P$. recondita & PUR 55742 & Leymus ambiguus & United States & JX533551 & $\mathrm{n} / \mathrm{a}$ \\
\hline$P$. recondita & PUR 25121 & Leymus arenarius & United States & JX533552 & $\mathrm{n} / \mathrm{a}$ \\
\hline$P$. recondita ${ }^{\mathrm{d}}$ & PUR 25280 & Elymus $\times$ vancouverensis & United States & JX533566 & JX533496 \\
\hline P. recondita ${ }^{\mathrm{d}}$ & PUR 25281 & $\begin{array}{l}\text { Leymus } \times \text { vancouverensis } \\
{[\text { mollis } \times \text { triticoides }]}\end{array}$ & Canada & JX533567 & JX533497 \\
\hline P. recondita & PUR 61943 & $\begin{array}{l}\text { Leymus } \times \text { vancouverensis } \\
{[\text { mollis } \times \text { triticoides }]}\end{array}$ & Canada & JX533565 & $\mathrm{n} / \mathrm{a}$ \\
\hline$P$. recondita & MIN 931119 & $\mathrm{n} / \mathrm{a}$ & United States & JX533590 & $\mathrm{n} / \mathrm{a}$ \\
\hline$P$. recondita ${ }^{\mathrm{d}}$ & MIN 931120 & S. cereale & United States & JX533569 & JX533499 \\
\hline$P$. recondita ${ }^{\mathrm{d}}$ & MIN 931121 & S. cereale & United States & JX533570 & JX533500 \\
\hline$P$. recondita ${ }^{\mathrm{d}}$ & ISR 9403 & S. montanum & Israel & JX533536 & JX533480 \\
\hline$P$. recondita ${ }^{\mathrm{d}}$ & MIN 931118 & Thalictrum sp. & United States & JX533568 & JX533498 \\
\hline$P$. recondita ${ }^{\mathrm{d}}$ & ISR 77081 & Triticum turgidum var. durum & Morocco & JX533538 & JX533482 \\
\hline$P$. recondita ${ }^{\mathrm{d}}$ & ISR 7784 & T. turgidum var. durum & Morocco & JX533529 & JX533473 \\
\hline$P$. recondita ${ }^{\mathrm{d}}$ & PUR F15509 (T) & S. cereale & France & JX533547 & JX533488 \\
\hline P. striiformis & PUR N 1249 & Hordeum jubatum & United States & HM057108g & $\mathrm{n} / \mathrm{a}$ \\
\hline P. striiformis & RS480 & T. aestivum & China & HM0571239 & $\mathrm{n} / \mathrm{a}$ \\
\hline P. striiformoides & BPI 0199096 & D. glomerata & China & HM057137g & $\mathrm{n} / \mathrm{a}$ \\
\hline P. striiformoides & PUR N5378 & D. glomerata & United States & HM057109g & $\mathrm{n} / \mathrm{a}$ \\
\hline P. subalpina ${ }^{\mathrm{d}, \mathrm{e}}$ & PUR F4065 (T) & E. caninus (in record) & Sweden & JX533544 & JX533486 \\
\hline P. triticina $a^{\mathrm{d}, \mathrm{e}}$ & (S) Reg.nr.F180131 (T) & T. vulgaris & Sweden & JX533571 & \\
\hline P. triticina & BP 88134 & Aegilops cylindrica & Hungary & HM147357f & $\mathrm{n} / \mathrm{a}$ \\
\hline P. triticina $^{\mathrm{d}}$ & ISR 99129 & A. speltoides & Israel & JX533572 & JX533507 \\
\hline P. triticina $^{\mathrm{d}}$ & ISR 99160-1 & A. speltoides & Israel & JX533573 & JX533508 \\
\hline P. triticina $^{\mathrm{d}}$ & Pt ISR1400 & A. speltoides & Israel & JX533577 & JX533506 \\
\hline P. triticina & PUR N1253 & E. repens & Canada & HM057146 & $\mathrm{n} / \mathrm{a}$ \\
\hline P. triticina & Pt AZB5 1 & T. aestivum & Azerbajian & $\mathrm{n} / \mathrm{a}$ & JX533502 \\
\hline P. triticina $^{\mathrm{d}}$ & Pt CHL8 53 & T. aestivum & Chile & JX533574 & JX533503 \\
\hline P. triticina & Pt Race 1 & T. aestivum & United States & $\mathrm{n} / \mathrm{a}$ & JX533509 \\
\hline P. triticina & Pt ARG9 3 & T. turgidum var. durum & Argentina & $\mathrm{n} / \mathrm{a}$ & JX533501 \\
\hline P. triticina $^{\mathrm{d}}$ & Pt ETH4114 7 & T. turgidum var. durum & Ethiopia & JX533575 & JX533504 \\
\hline P. triticina $^{\mathrm{d}}$ & Pt F2 2 & T. turgidum var. durum & France & JX533576 & JX533505 \\
\hline P. wyomensis ${ }^{\mathrm{d}}$ & PUR 24906 (T) & Agropyron sp. (redetermined) & United States & JX533541 & $\mathrm{n} / \mathrm{a}$ \\
\hline Uromyces dactylidis (outgroup) & DAOM 216236 & D. glomerata & Hungary & HM057148g & $\mathrm{n} / \mathrm{a}$ \\
\hline
\end{tabular}


specimens. As a result, the data set for ITS included a broader range of taxa than for EF1- $\alpha$.

Alignment and phylogenies. The alignment of 110 5.8S-ITS2 sequences resulted in 544 characters, among which 146 characters are parsimony-informative; 351 most parsimonious trees were retained of length $=614, \mathrm{CI}=0.467, \mathrm{RI}=0.831, \mathrm{RC}=0.388, \mathrm{HI}=$ 0.533 , and G-fit $=-99.908$ (Fig. 2). The majority of the $P$. recondita sensu lato samples collected on Aegilops, Elymus, and

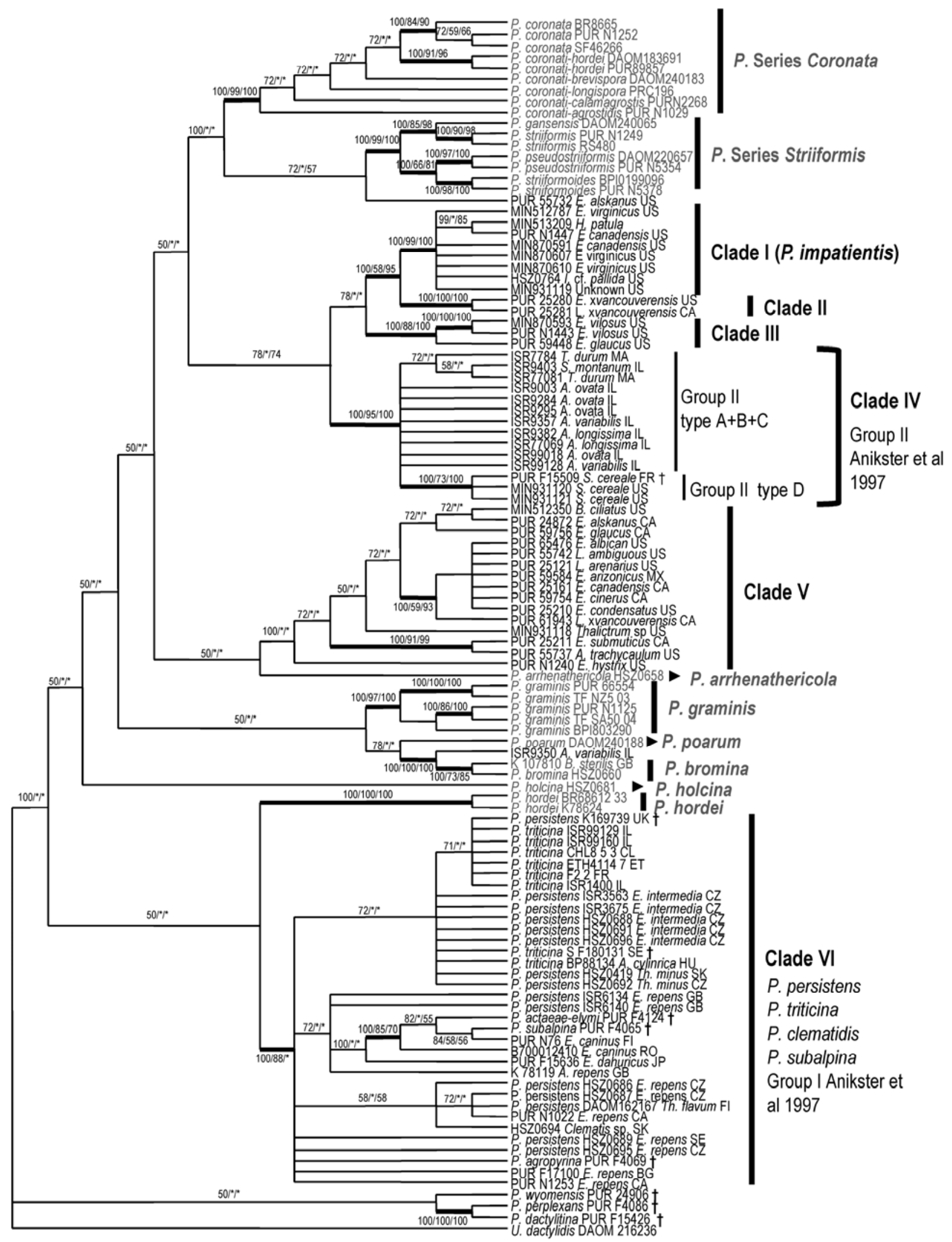

Fig. 2. Of 351 most parsimonious trees, 1 tree based on $5.8 \mathrm{~S}$-internal transcribed spacer 2 rooted with Uromyces dactylidis. Values on the branches are majority rule frequencies/parsimony bootstrapping values/Bayesian posterior probabilities; ${ }^{*}$ indicates value lower than $50 \%$. Branches with parsimony bootstrapping value $>50$ and Bayesian posterior probability $>70$ are in bold. Puccinia recondita samples labeled with voucher number, host, and country code, other species labeled with species name and voucher number. Type specimens and the samples in $P$. recondita complex were highlighted in black fonts, others in gray fonts; $\dagger$ indicates type specimens. 
Triticum spp. were distributed in six major clades: clades I, II, and III were strongly supported individually and as a group received some support $(78 / * / 74)$ (majority rule frequencies/parsimony bootstrapping values/Bayesian posterior probabilities) for a close relationship with clade IV, which was consistent with $P$. recondita group II from Anikster et al. (1). Clade V had low statistical support and did not show a strong relationship to any other clade. $P$. persistens and $P$. triticina were intermixed in one large, strongly supported clade VI, which also included five specimens identified as $P$. recondita: two on Elymus caninus (PUR N76 and B700012410), two on E. repens (PUR F17100 and PUR N1253), and one on E. dahuricus (PUR F15636). Five type specimens K(M): 169739 (P. persistens), (S) Reg.nr.F180131 (P. triticina), PUR F4069 (P. agropyrina), PUR F4124 (P. actaeae-elymi), and PUR F4065 ( $P$. subalpina) were included in the $P$. persistens $/ P$. triticina clade. The type specimen of $P$. recondita formed a monophyletic group along with the other two rye leaf rust collections in group II type D of Anikster et al. (1). Type specimens of $P$. perplexans and $P$. dactylidina were closely related but not robustly connected with any other samples while the type specimen of $P$. wyomensis appeared as an orphan lineage in the phylogeny.

The alignment of $58 \mathrm{EF} 1-\alpha$ sequences resulted in 556 characters, among which 207 characters were parsimony-informative; 57,624 most parsimonious trees were retained of length $=700, \mathrm{CI}$ $=0.5811, \mathrm{RI}=0.884, \mathrm{RC}=0.514, \mathrm{HI}=0.419$, and $\mathrm{G}-\mathrm{fit}=$ -149.918 (Fig. 3). Shallow (terminal) groupings of taxa were generally congruent with those of the rDNA tree in Figure 2; however, the EF1- $\alpha$ tree provided better resolution. Clades I, II, and III were the same as in the rDNA tree. Clade IV was separated into three subclades (clade IV-1, IV-2, and IV-3), with the type specimen of $P$. perplexans grouped in subclade IV-2 and the type of $P$. recondita in subclade IV-3. Clade VI was separated into three subclades: VI-1, VI-2, and VI-3. The type specimens of P. actaeaeelymi and $P$. subalpina grouped in subclade VI-3.

Sequences for 54 samples with both rDNA and EF1- $\alpha$ data available were concatenated. In addition, eight samples with only one sequence region were added to that matrix. Among these eight samples, seven samples have only rDNA and one sample has only EF1- $\alpha$ locus. These five type specimens were K(M): 169739(T), PUR F4069(T), PUR F15426(T), PUR 24906(T), and (S)Reg.nr.F 180131(T); two P. recondita on Bromus ciliates (MIN 512350) and E. alskanus (PUR 24872), respectively, from clade V; and one $P$. recondita on E. semicostatus (PUR F16132) from clade VI-2. The absent sequences were coded as missing data, which does not limit the accuracy of phylogenetic placement of those eight taxa (47). The concatenated alignment resulted in 1,089 characters for 62 taxa, among which 287 characters are parsimony-informative; 34,866 most parsimonious trees were retained of length $=1036, \mathrm{CI}$ $=0.547, \mathrm{RI}=0.860, \mathrm{RC}=0.471, \mathrm{HI}=0.453$, and $\mathrm{G}-\mathrm{fit}=-201.739$ (Fig. 4). Compared with the individual gene trees, the tree based on the combined loci revealed better support for the close relationship among clades VI-1, VI-2, and VI-3. A preliminary analysis indicated that $U$. dactylidis was not an appropriate outgroup for this species complex for the EF1- $\alpha$ gene; therefore, the EF1- $\alpha$ and combined data analyses were midpoint-rooted instead. Data matrices and trees were submitted to TreeBASE, study number S13719.

\section{Discussion}

The classification of brown leaf rust fungi on cereals and grasses has experienced a long history of controversy and uncertainty due to the reduced morphological characteristics available for taxonomy and the limitation of conducting interfertility experiments. Analyses of DNA sequences have proven effective for elucidating phylogenetic relationships among species in other fungi $(24,43)$ but had not yet been adopted for examining the $P$. recondita complex. Our present study is an initial step toward clarifying the intertwined relationships within this species complex. Phylogenetic trees recovered multiple phylogenetic lineages which are congruent with the existing classifications based on interfertility, host specificity, and morphology but also displayed a number of lineages that can be potential new species.

Rye leaf rust fungus (P. recondita sensu stricto). The name $P$. recondita was originally applied to a rust specimen on rye collected by Roberge (11). A hundred years later, Cummins and Caldwell (8) used the name to replace $P$. rubigo-vera, the name of a broader species concept commonly referred to as brown leaf rust fungi on cereals and grasses. Since then, the name $P$. recondita has conveyed different species concepts: in a general sense, $P$. recondita sensu lato includes the brown leaf rust fungi on various cereals and grasses (7) whereas, in the restricted concept, $P$. recondita sensu stricto only encompasses the taxon on rye and a few other host species $(44,45)$. Despite the long taxonomic history and extensive research assessing host specialization within $P$. recondita sensu lato using infection experiments $(12,28)$ and the recognition of the wheat leaf rust fungus ( $P$. triticina) as distinct from the rye leaf rust fungus based on infection structures and morphology $(14,39)$, there have been only a few studies focused on determining the exact host range of $P$. recondita sensu stricto. According to Urban and his colleagues, the natural hosts of $P$. recondita sensu stricto include Secale and Aegilops spp. (45). Based on genome size, host range, and interfertility, Anikster et al. (1) separated $P$. recondita on wheat, rye, and Aegilops spp. into two groups: group I infecting cultivated wheat and wild emmer wheat and group II infecting rye and Aegilops spp. Group II was further divided into four types, with type D restricted to rye. In this study, the phylogenetic trees based on EF1- $\alpha$ (Fig. 3) and combined rDNA (5.8ITS2) and EF1- $\alpha$ data (Fig. 4) partly supported the separation of group II into different types. A strongly supported clade IV was further divided into three subclades: subclade IV-1, with collections from A. variabilis, A. longissima, and T. durum, was consistent with group II type A and C from Anikster et al. (1) with support 100/97/100; and subclade IV-2, with collections from $A$. ovata, was consistent with type B, with support of 100/52/91. Subclade IV-2 also included the type specimens of $P$. perplexans (PUR F4086) and $P$. dactylidina (PUR F15426). Subclade IV-3 with, collections from $S$. montanum and $S$. cereale, was consistent with group II type D of Anikster et al. (1), with support of 100/100/100. The type specimen of $P$. recondita (PUR F15509) also was placed in this subclade. Given the strong support for three subclades, three corresponding phylogenetic species might be recognized. For the first species (subclade IV-1) that corresponds to group II types A and C from Anikster et al. (1), none of the type specimens sampled were included from which to select a potentially valid name. For the second species (subclade IV-2) that corresponds to group II type B of Anikster et al. (1), the type specimens of both $P$. perplexans and $P$. dactylidina were present. $P$. perplexans described on A. pratensis (uredinial or telial stage) and $R$. acris (aecial stage) by Plowright (34) predates $P$. dactylidina by Bubák (5); therefore, the name $P$. perplexans is potentially the duly legitimate name for this lineage. Multiple cross fertilization studies of brown rust indicated that $P$. recondita on A. ovata (also present in this clade) with an alternate host in the genus Echium (family Boraginaceae), was genetically isolated from other forms $(1,12)$ and merited a consideration as distinct species. Comparing the results from our DNA sequence analyses with the fertilization experiments, it seems likely that the lineage on $A$. ovata could be considered as a distinct species, with uredinial or telial hosts that include A. ovata, A. pratensis, and Dactylis glomerata and aecial hosts in both the families Ranunculaceae and Boraginaceae. A case like this is not common but yet possible, such as the observation by Marková and Urban (30) that the aecial hosts of $P$. persistens subsp. agropyri are not only in the family Ranunculaceae but also in the family Boraginaceae in Europe. The third species (subclade IV-3), corresponding to group II type D of Anikster et al. (1), appears as the authentic $P$. recondita sensu stricto. The uredinial or telial hosts of $P$. recondita sensu stricto are very likely limited to Secale spp., with the aecial host limited to the family Boraginaceae, as discussed in previous studies $(12,28)$. 
Wheat leaf rust fungus. The taxonomic distinction of leaf rust fungus on wheat from the other leaf rust fungi has also been controversial. Leaf rust on wheat has been treated as a specialized form of $P$. rubigo-vera (or $P$. recondita), as a distinct species, and as a specialized form of $P$. persistens. The wheat leaf rust fungus was originally observed as a host-specific form of $P$. rubigo-vera by Eriksson and Henning (15). This classification was accepted by Carleton (6) and Mains (28). When the name of P. rubigo-vera was replaced with $P$. recondita, the wheat leaf rust fungus was then considered as a specialized form of $P$. recondita by Cummins (7).

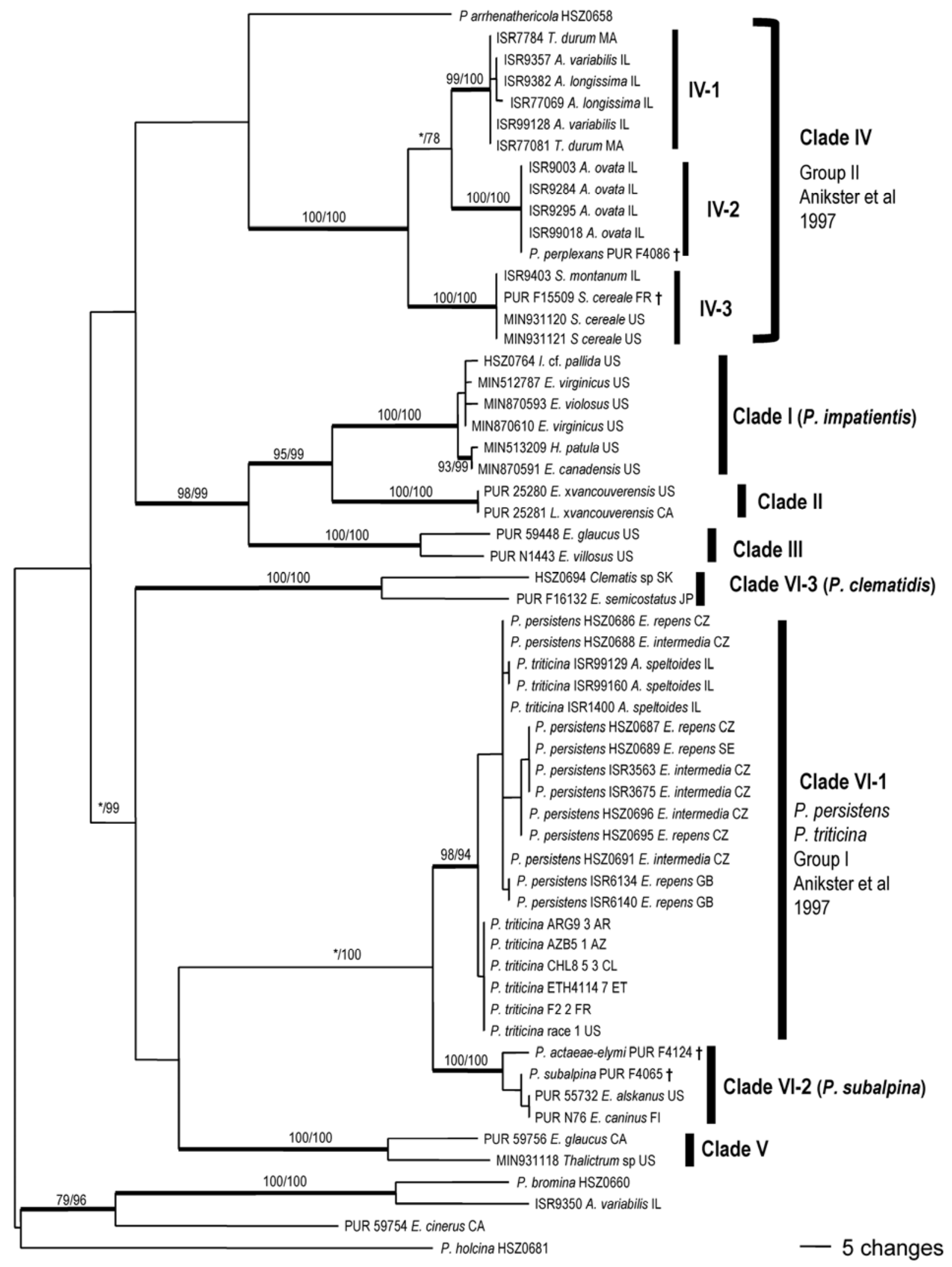

Fig. 3. Of 57,624 most parsimonious midpoint-rooted phylograms, 1 phylogram based on elongation factor 1- $\alpha$ partial DNA sequences. Values on branches are parsimony bootstrapping/Bayesian posterior probability. Majority frequencies for all present branches are $100 \%$. Puccinia recondita samples labeled with voucher number, host, and country code; other species labeled with species name and voucher number; $†$ indicates type specimens. 
Eriksson (14) conducted an extensive morphological study and concluded that the leaf rust fungus on wheat was a distinct species; namely, $P$. triticina. Other researchers also separated the wheat leaf rust fungus from the rye leaf rust fungus based on infection experiments (12), morphology of urediniospore germlings $(32,33,41)$, and morphology of sori and spores (39).

Marková and Urban (30) treated the wheat leaf rust fungus as a specialized form of $P$. persistens (16,21-23). P. persistens was described on E. repens (recorded as T. repens on the type specimen K(M): 169739) by Plowright (35). The classification of Marková and Urban that separated the species into three subspecies ( $P$. persistens subspp. persistens, triticina, and agropyri) was based on minute differences in the shape of teliospores and host ranges. $P$. persistens subsp. persistens has broader teliospores (brachysporic, the ratio of length to lower cell width of 2 to 3 ), whereas the other two subspecies have longer and narrower spores (dolichosporic, ratio of 3 to 4). Among the three subspecies, P. persistens subsp. triticina has the narrowest host range, on wheat (uredinial or telial host), with alternate hosts in Thalictrum spp. The names $P$. triticina and $P$. agropyrina were treated as synonyms of $P$. persistens subsp. triticina and $P$. persistens subsp. agropyri, respectively (30). Although more recent DNA sequence analysis based on rDNA ITS and $28 \mathrm{~S}$ revealed potential separation of three subspecies (L. S. Szabo, unpublished), our analyses, based on 5.8S-ITS2 and EF1 partial sequences, was not able to clearly separate them (Figs. 24). Marková and Urban (30) also referred to the observation of Jackson and Mains $(18,29)$ of the close relationship between $P$. triticina and $P$. persistens, and questioned the level of closeness of the two species. Based on the loci sampled in our study, it appears that the two species are barely separable. Although $P$. triticina isolates formed a subclade nested in the clade of $P$. persistens and $P$. triticina, the statistical supports were very low $(50 / * / 59$; Fig. 4) and the type specimen of $P$. persistens (with only $5.8 \mathrm{~S}$ and ITS2) is also located in this subclade of $P$. triticina. However, sequence data from nine additional hypervariable loci clearly separated $P$. persistens and $P$. triticina (M. Liu and J. Kolmer, unpublished data). We speculate that the divergence among these lineages occurred recently and, therefore,

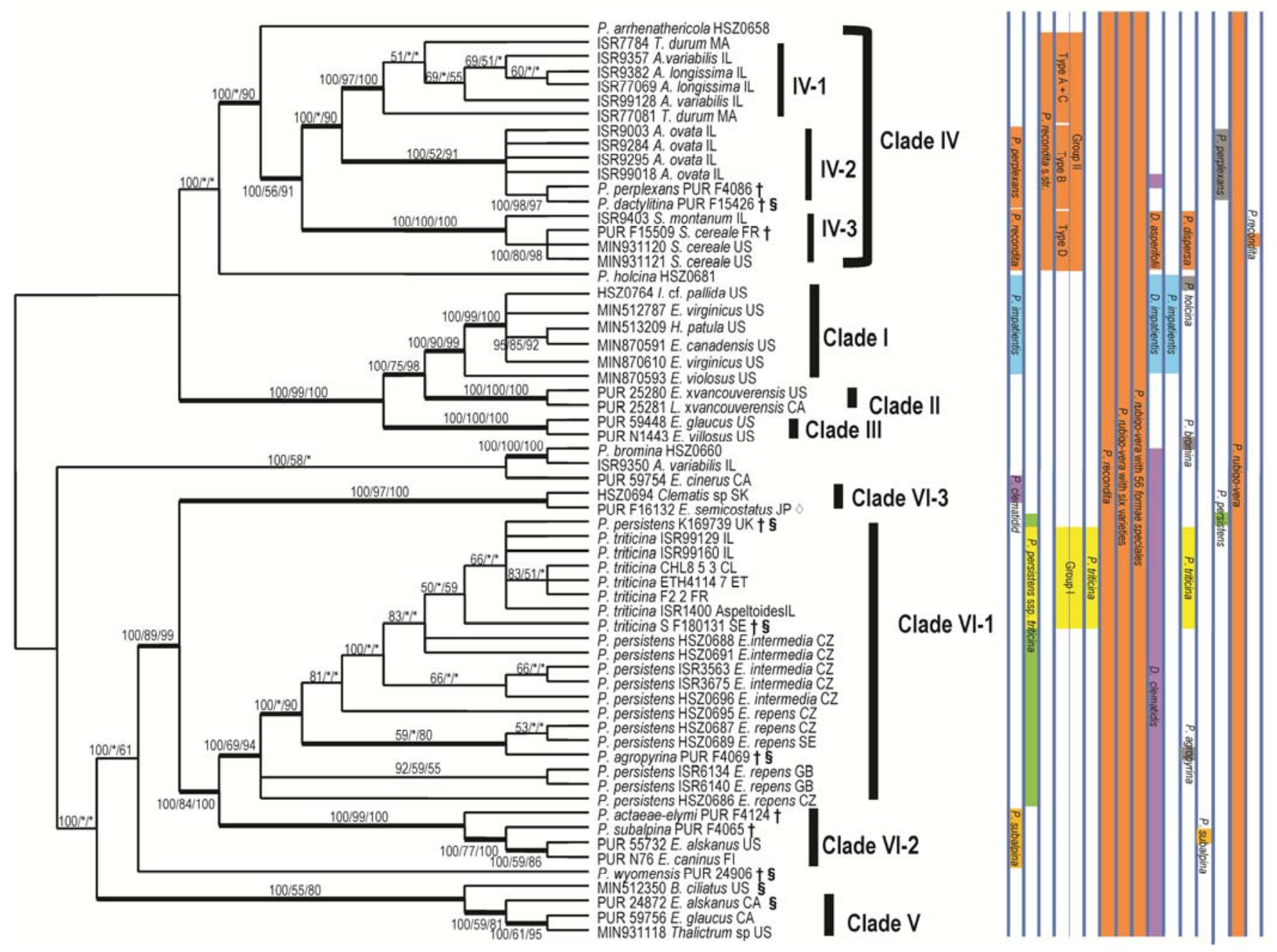

Fig. 4. Majority consensus of 34,866 most parsimonious trees based on combined 5.8-internal transcribed spacer2 and elongation factor $1-\alpha($ EF1- $\alpha$ ) loci midpoint rooted. Puccinia recondita samples labeled with voucher number, host, and country code; other species labeled with species name and voucher number. Values on the branches are majority rule frequencies/parsimony bootstrapping values/Bayesian posterior probabilities; ${ }^{*}$ indicates value lower than $50 \%$. Branches with parsimony bootstrapping value $>50$ and Bayesian posterior probability $>70$ are in bold; $\dagger$ indicates type specimens; $\S$ samples have only ribosomal DNA (rDNA) sequences, not EF1- $\alpha$; $\diamond$ indicates one sample has only EF1- $\alpha$ sequence, not rDNA. In the immediate column to the right of the tree are listed the proposed valid names for selected clades from this study. These proposed names are compared with previous names accepted by researchers listed at the top of the columns. Except for gray color, each of the other colors represents equivalent taxa. 
the level of genetic variation in relatively conserved regions is low.

$P$. recondita on Elymus spp. The samples of $P$. recondita sensu lato on Elymus spp. did not form a monophyletic group. In addition to the isolates on $E$. repens and E. intermedia, which were located in the $P$. persistens and $P$. triticina clade, other isolates were distributed into six clades (I, II, III, V, VI-2, and VI-3). Each one received strong statistical support, suggesting six distinct phylogenetic species. Here, we discuss the potentially correct taxonomic names for clades I, VI-2, and VI-3 based on associated type specimens, host range, and literature research. For clades II, III, and V, further evidence based on either aecial hosts or type specimens are needed to clarify their identities.

Clade I, comprising collections from E. canadensis, E. violosus, E. virginicus, Hystrix spp., and Impatiens cf. pallida, is consistent with the concept of P. impatientis (Schwein.) Arthur (2), although the taxonomic placement of this taxon has changed several times. The aecial state on Impatiens maculata (Impatientis maculatae) was originally described as Aecidium impatientis by Schweinitz (40). Arthur (2) successfully made the connection between the rust from Impatiens aurea with $P$. rubigo-vera on E. virginicus and, therefore, proposed to move the species into the genus Puccinia. However, in a revision of genus Dicaeoma, Arthur and Fromme (4) included the taxon under the name Dicaeoma impatientis (Schw.) Arthur \& Fromme. The hosts were listed as I. biflora Walter and I. pallida Nutt. as aecial hosts, and the following species as uredinial or telial hosts: Agrostis alba, A. hyemalis, A. perennans, Agrostis sp., Cinna arundinacea, C. latifolia, E. canadensis, E. striatus, E. virginicus, Hordeum jubatum, and Hystrix hystrix. Mains (28) listed $P$. impatientis as a synonym of the forma specialis $P$. rubigovera impatientis; meanwhile, he accepted another four formae speciales of brown leaf rust fungi with aecia on species of Impatiens based on slightly different infection profiles, which were $P$. rubigo-vera similis, $P$. rubigo-vera graminicola, $P$. rubigo-vera singularis, and one unnamed forma specialis infecting $H$. hystrix. $P$. rubigo-vera impatientis had compatible infection with $E$. canadensis and E. virginicus, which is consistent with the host range of clade I. Because the lineage is strongly supported as monophyletic (Figs. 2 and 4,100/99/100; Fig. 3, 100/90/99), it is reasonable to propose this taxon as a species. Because the name $P$. impatientis by Arthur was the earliest published, it should be the appropriate name for this phylogenetic species.

Clade VI-2 includes four specimens: [rephrased for clarity] one on $E$. caninus, one on E. alaskanus, and the two type specimens $P$. actaeae-elymi (PUR F4124) and $P$. subalpina (PUR F4065). Mayor (31) made the connection between the rust on E. europaeus (current name $=$ Hordelymus europaeus (L.) Harz) with the aecial host Actaea spicata L., and named the rust as P. actaeae-elymi Mayor. No record was found for P. subalpina in the MycoBank database (Stalpers, J. de Cock, A. Robert, V. Vu, D and Ir. Ammar Ben Hadj Amor, http://www.mycobank.org/Biolomics.aspx?Table=Myco bank\&Page $=200 \&$ ViewMode $=$ Basic) or in Index Fungorum (D. F. Farr and A. Y. Rossman, Fungal Databases, Systematic Mycology and Microbiology Laboratory, United States Department of Agriculture-Agricultural Research Service, http://www.index fungorum.org/Names/Names.asp). Based on the notes on the type specimen, the name was erected by G. v. Lagerheim in 1897 and published in Sydow's exsiccati [Sydow, uredineen 1384]. The host of the type specimen was originally recorded as [Tritrici] anini, later corrected as E. caninus by anonymous. Because $P$. subalpina Lagerh. is an earlier, validly published name than P. actaeae-elymi, it is the correct name for this lineage.

Clade VI-3 includes two specimens, one on E. semicostatus from Japan and the other on Clematis sp. Arthur and Fromme (4) adopted $D$. clematidis (later renamed $P$. clematidis) to include all the $P$. rubigo-vera forms with aecial hosts in the family Ranunculaceae. Saccardo (38) recorded P. clematidis-agropyri Ellis \& Everh. with the aecial host of Clematis vitalba and the uredinial or telial host of Agropyron[um] glaucum (current name = Thinopyrum intermedium subsp. intermedium). Mains (28) separated P. rubigo- vera on Clematis spp. into 11 formae speciales by infection profiles, among which 8 formae speciales have compatible or moderate infection reactions with Elymus spp. (E. arkansanus, E. canadensis, E. condensatus [current name = Leymus condensatus], E. glaucus, and E. virginicus), Hordeum spp. (Hordeum jubatum and H. gussoneanum), and Hystrix hystrix. One forma specialis was compatible with Agropyron tenerum (current name $=E$. trachycaulus subsp. trachycaulus), while the final two formae speciales were compatible with Bromus spp. E. semicostus was not included in their infection experiment. Arthur and Fromme's (4) concept is too broad to fit this phylogenetic lineage. The concept of Ellis and Everhardt (38) and any of the formae speciales recognized by Mains (28) should be further evaluated by including more samples on the host $T$. intermedium subsp. intermedium and the range of hosts tested by Mains (28) for compatibility.

With the combination of molecular phylogenetic tools and biology, this study sheds light on the phylogenetic diversity of the brown rust fungi on Elymus spp. and the concepts of $P$. recondita sensu stricto and the relationship between $P$. tritcina and $P$. persistens. For $P$. recondita sensu lato, there are still numerous questions to address in terms of how many distinct species should be recognized and how they are related. The analyses and interpretations presented here can serve as a foundation for future targeted studies addressing these questions.

\section{Acknowledgments}

We thank all the herbaria for kindly lending specimens for our study: Botanischer Garten und Botanisches Museum Berlin-Dahlem, Zentraleinrichtung der Freien Universität Berlin, Berlin; Hungarian Natural History Museum, Budapest; U.S. National Fungus Collections, Beltsville, MD; National Botanic Garden of Belgium, Meise, Belgium; Agriculture and Agri-Food Canada, Ottawa, Ontario, Canada; Royal Botanic Gardens, Kew, England, UK; J. F. Bell Museum of Natural History, University of Minnesota, St. Paul; Charles University in Prague, Praha, Czech Republic; Purdue University, West Lafayette, IN; one sample held by S. Hambleton as DNA voucher only; and the Swedish Museum of Natural History, Stockholm; and two anonymous reviewers and the editor for valuable suggestions to improve this manuscript.

\section{Literature Cited}

1. Anikster, Y., Bushnell, W. R., Roelfs, A. P., Eilam, T., and Manisterski, J. 1997. Puccinia recondita causing leaf rust on cultivated wheats, wild wheats, and rye. Can. J. Bot. 75:2082-2096.

2. Arthur, J. C. 1903. Cultures of Uredineae in 1902. Bot. Gaz. 35:19.

3. Arthur, J. C. 1934. Manual of the Rusts in United States and Canada. Purdue Research Foundation, Layfayette, IN.

4. Arthur, J. C., and Fromme, F. D. 1920. Uredinales, Aecidiaceae, Dicaeoma on Poaceae. N. Am. Flora 7:327-338.

5. Bubák, F. 1905. Ann. Mycol. 3:219.

6. Carleton, M. A. 1899. Cereal rusts of the United states: A physiological investigation. U.S. Dep. Agric. Div. Veg. Physiol. Pathol. Bull. No. 16.

7. Cummins, G. B. 1971. The Rust Fungi of Cereals, Grasses and Bamboos Springer-Verlag, New York, Heidelberg, Berlin.

8. Cummins, G. B., and Caldwell, R. M. 1956. The validity of binomials in the leaf rust fungus complex of cereals and grasses. Phytopathology 46:81-82.

9. de Bary, A. 1866. Neue Untersuchungen über Uredineen. Monatsber. K. reuss. Akad. Wiss, Berlin.

10. De Candolle, A. P. 1815. Uredo rubigo-vera. Page 83 in: Flore française, ou, Descriptions succinctes de toutes les plantes qui croissent naturellement en France: Disposées selon une nouvelle méthode d'analyse, et précédées par un exposé des principes élémentaires de la botanique. J. B. P. A. M. Lamarck and A. P. Candolle, eds. Desray, Paris.

11. Desmazières, J. B. H. J. 1857. Vingt-quatrième notice sur les plantes cryptogames récemment découvertes en France. Bull. Soc. Bot. France 4:798.

12. d'Oliveira, B. D., and Samborski, D. J. 1966. Aecial stage of Puccinia recondita on Ranunculaceae and Boraginaceae in Portugal. Pages 133-150 in: Proc. First Eur. Brown Rust Conf. Cereal Rust Conf. R. C. F. Macer, and M. S. Wolfe, eds. Cambridge, UK.

13. Eriksson, J. 1894. Uber die specializierung des parasitismus bei den Getreiderostpilzen. Ber. Dtsch. Bot. Ges. 12:292-331.

14. Eriksson, J. 1899. Nouvelles études sur la rouille brune des cécéales. Ann. Sci. Nat. Bot. Sér. 8:241-288.

15. Eriksson, J., and Henning, E. 1894. Die Hauptresultate einer neuen Untersuchung über die Getreiderostpilze. Z. Pflanzenkrankh. 4:197-203, 257-262.

16. Gäumann, E. A. 1936. Über den Formenkreis der Puccinia persistens Plowright. Ber. Schweiz. Bot. Ges. 46:229-247.

17. Hall, T. A. 1999. BioEdit: A user-friendly biological sequence alignment 
editor and analysis program for Windows 95/98/NT Nucleic Acids Symp. Ser. 41:95-98.

18. Jackson, H. S., and Mains, E. B. 1921. Aecidial stage of the orange leaf rust of wheat, Puccinia triticina Eriks. J. Agric. Res. 22:151-172.

19. Jiang, J., B. Friebe, and B. S. Gill. 1993. Recent advances in alien gene transfer in wheat. Euphytica 73:199-212. Online publication. doi:10.1007/ bf00036700

20. Katoh, K., Kuma, K.-I., Toh, H., and Miyata, T. 2005. MAFFT version 5: Improvement in accuracy of multiple sequence alignment. Nucleic Acids Res. 33:511-518.

21. Khokhryakov, M. K. 1940. K voprosam sistematiki ržavčiny na pšenic. Tezisy nauč. Sess. Leningrad. selsk. chozjaj. Inst. 1940:117.

22. Khokhryakov, M. K. 1941. Specializacija vidov ržaviny chlebnych zlakov v nečernozemnoj polose jevropejskoj časti SSSR. Věstn. zašč. Rast. 1:116125.

23. Khokhryakov, M. K. 1955. O vide u gribov. Bot. Z. 40:33-45.

24. Kohn, L. M. 2005. Mechanisms of fungal speciation. Annu. Rev. Phytopathol. 43:279-308.

25. Liu, M., and Hambleton, S. 2010. Taxonomic study of stripe rust, Puccinia striiformis sensu lato, based on molecular and morphological evidence. Fungal Biol. 114:881-899.

26. Liu, M., and Hambleton, S. 2013. Laying the foundation for a taxonomic review of Puccinia coronata s.l. in a phylogenetic context. Mycol. Prog. 12:63-89.

27. Liu, M., and Hambleton, S. 2012. Puccinia chunjii, a close relative of the cereal stem rusts revealed by molecular phylogeny and morphological study. Mycologia 104:1056-1067.

28. Mains, E. B. 1933. Host specialization in the leaf rust of grasses, Puccinia rubigo-vera. Pap. Mich. Acad. Sci. Arts Lett. 17:289-394.

29. Mains, E. B., and Jackson, H. S. 1926. Physiologic specialization in the leaf rust of wheat, Puccinia triticina Eriks. Phytopathology 16:89-120.

30. Marková, J., and Urban, Z. 1998. The rust fungi of grasses in Europe. 6. Puccinia persistens Plow., P. perplexans Plow., and P. elymi Westend. Acta Univ. Carol. Biol. 41:329-402.

31. Mayor, E. 1911. Recherches expérimentales sur quelques Urédinées hétérö̈ques. Ann. Mycol. 9:355-362.

32. Niks, R. E. 1986. Variation of mycelial morphology between species and formae speciales of rust fungi of cereals and grasses. Can. J. Bot. 64:29762983.

33. Niks, R. E., and Dekens, R. G. 1987. Histological studies on the infection of triticale, wheat and rye by Puccinia recondita f. sp. tritici and $P$. recondita f. sp. recondita. Euphytica 36:275-285. Online publication. doi:10.1007/ bf00730674

34. Plowright, C. B. 1885. On the life history of certain British heteroecismal Uredines (The Ranunculi Aecidia and Puccinia schoelerianna). Q. J. Microsc. Sci. 25:151-172.

35. Plowright, C. B. 1889. A Monograph of the British Uredineae and Ustilagineae. Kegan Paul, Trench \& Co., I, Paternoster Square, London.

36. Posada, D., and Crandall, K. A. 1998. Modeltest: Testing the model of DNA substitution. Bioinformatics 14:817-818.

37. Ronquist, F., Teslenko, M., van der Mark, P., Ayres, D. L., Darling, A., Höhna, S., Larget, B., Liu, L., Suchard, M. A., and Huelsenbeck, J. P. 2012. MrBayes 3.2: Efficient Bayesian phylogenetic inference and model choice across a large model space. Syst. Biol. Online publication. doi:10.1093/ sysbio/sys029

38. Saccardo, P. A. 1905. Puccinia (clematidis-)agropyri Ell. et Everh. Sylloge fungorum omnium hususque cognitorum XVII:465.

39. Savile, D. B. O. 1984. Taxonomy of the cereal rust fungi. Pages 79-111. in: The Cereal Rusts: Origins, Specificity, Structure, and Physiology W. R. Bushnell, and A. P. Roelfs, eds. Academic Press, New York

40. Schweinitz, L. D. V. 1822. Synopsis fungorum Carolinae superioris: Secundum observations. No. 442. C. F. Schwägrichen, ed. Leipzig, Germany.

41. Swertz, C. A. 1994. Morphology of germlings of urediniospores and its value for the identification and classification of grass rust fungi. Stud Mycol. 36:1-157.

42. Swofford, D. L. 1998. PAUP*. Phylogenetic Analysis Using Parsimony (*and other methods), version 4 (computer program). Sinauer Associates, Sunderland, MA

43. Taylor, J. W., Jacobson, D. J., Kroken, S., Kasuga, T., Geiser, D. M. Hibbett, D. S., and Fisher, M. C. 2000. Phylogenetic species recognition and species concepts in fungi. Fungal Genet. Biol. 31:21-32.

44. Urban, Z. 1966. On the taxonomy of Puccinia recondita Rob. ex Desm. s.1. Ceská Mykol. 20:38-44.

45. Urban, Z. and Marková, J. 1996. The rust fungi of grasses in Europe. 4 Puccinia recondita Rob. ex Desm. s. str. Acta Univ. Carol. Biol. 37:93-147.

46. Urban, Z., and Marková, J. 1999. The rust fungi of grasses in Europe. 8. Puccinia bromina Eriks. Acta Univ. Carol. Biol. 43:119-134.

47. Wiens, J. J. 2003. Missing data, incomplete taxa, and phylogenetic accuracy. Syst. Biol. 52:528-538

48. Winter, G. 1884. 330. P. rubigo-vera (DC). Rabenhorst's KryptogamenFlora 1:217. 